\title{
Local and Global Flatness in Cosmology
}

\section{Rainer Burghardt}

A-2061 Obritz 246, Obritz, Austria

Email:arg@aon.at

How to cite this paper: Burghardt, R. (2019) Local and Global Flatness in Cosmology. Journal of Modern Physics, 10, 1439-1453.

https://doi.org/10.4236/jmp.2019.1012096

Received: August 22, 2019

Accepted: November 2, 2019

Published: November 5, 2019

Copyright $\odot 2019$ by author(s) and Scientific Research Publishing Inc. This work is licensed under the Creative Commons Attribution International License (CC BY 4.0).

http://creativecommons.org/licenses/by/4.0/

\begin{abstract}
We raise the question of how the curvature parameter $k$ is related to the curvature of the universe. We also show that, for a cosmological model that can be interpreted geometrically as a pseudo-hypersphere with a time-dependent radius, the Einstein field equations are not sufficient to fully describe the model. In addition, the differential equation system of Bianchi identities is required to describe the temporal evolution of the universe. We discuss the facts using the example of the de Sitter universe, the subluminal universe and the $R_{h}=c t$ model by Melia. In particular, we discuss the formal differences between the two latter models and claim that both models are identical. We also examine the possibility of introducing non-comoving coordinates.
\end{abstract}

\section{Keywords}

Curvature Parameter, Bianchi Identities, de Sitter Cosmos, Subluminal Cosmos, $R_{h}=c t$ Cosmos, Geometric Horizon

\section{Introduction}

In many papers on expanding cosmological models, the topic is introduced with findings on the curvature parameter $k$. An expanding model is based on the metric in the canonical form

$$
\mathrm{d} s^{2}=\frac{1}{1-k \frac{r^{\prime 2}}{R^{2}}} \mathrm{~d} r^{\prime 2}+r^{\prime 2} \mathrm{~d} \Omega^{2}-\mathrm{d} t^{\prime 2} .
$$

Here, $r^{\prime}$ is the comoving radial coordinate of an observer participating in an expanding motion and $\Omega$ the solid angle. $t^{\prime}$ is the cosmic time, which applies equally to all comoving observers and, at the same time, is the proper time of these observers. For $k=1$ the underlying space should be positively curved and closed. For $k=0$ the space is described as flat and $k=-1$ negatively curved. The two latter universes are open, they exhibit infinite extension. 
In an earlier paper [1], we showed that $k=0$ does not necessarily mean that the universe described by the line element (1.1) is flat. We discuss this problem once again in Sec. 2. Sec. 3 deals extensively with the two versions of the de Sitter universe and its inconsistencies. In Sec. 4, we extend the considerations to the subluminal universe and to Melia's model in Sec. 5. We also discuss the 3-dimensional Ricci scalar and how meaningful the relation ${ }^{3} R=0$ is for $k=0$. In Sec. 6, we explore the possibilities of finding coordinate systems for non-comoving systems.

Furthermore, we will use the following variables: $R$ radius of the universe, $K$ scale factor, $H$ Hubble parameter, $B, C, U$ curvature quantities, $D$ tidal forces.

\section{The Curvature Parameter}

In our paper [1], we examined in detail the free fall in the Schwarzschild field, with the intention of extending the associated methods to expanding cosmological models. With the transformation

$$
\sqrt{\frac{2 M}{r}}=\frac{r}{R}
$$

the Schwarzschild metric

$$
\mathrm{d} s^{2}=\frac{1}{1-\frac{2 M}{r}} \mathrm{~d} r^{2}+r^{2} \mathrm{~d} \Omega^{2}-\left(1-\frac{2 M}{r}\right) \mathrm{d} t^{2}
$$

can be converted into the canonical form

$$
\mathrm{d} s^{2}=\frac{1}{1-\frac{r^{2}}{R^{2}}} \mathrm{~d} r^{2}+r^{2} \mathrm{~d} \Omega^{2}-\left(1-\frac{r^{2}}{\mathrm{R}^{2}}\right) \mathrm{d} t^{2} .
$$

Here, $R=R(r)$ is half the radius of curvature of the Schwarzschild parabola and according to $(2.1)$ has the validity range $R=[2 M, \infty]$. At the waist of Flamm's paraboloid one has $R=2 M$ and this marks the event horizon.

Comparison with (1.1) shows that the curvature parameter of the metric is $k=1$, Schwarzschild geometry thus builds on a positively curved space. Furthermore, (2.3) formally corresponds to the line element of the de Sitter universe. We will build on this.

Lemaitre used a coordinate transformation to transform the Schwarzschild metric into the form

$$
\mathrm{d} s^{2}=\mathrm{K}^{2}\left[\mathrm{~d} r^{\prime 2}+\mathrm{R}^{2} \mathrm{~d} \Omega^{2}\right]-\mathrm{d} t^{\prime 2}, \quad K=\frac{r}{\mathrm{R}} .
$$

As in the cosmological models, $K$ is referred to here as a scale factor. The line element is of type $k=0$. The new coordinate system $\left(i^{\prime}\right)$ accompanies a free-falling observer family. $t^{\prime}$ is the common time for all observers and $r^{\prime}$ the comoving radial coordinate. From the metric (2.4), we learn that $g_{4^{\prime} 4^{\prime}}=1$. This means that there is no gravity present in this system. To get more insight 
into the problem, we should remember the following: Observers hover in a closed elevator. Since they are not familiar with their environment, they consider themselves motionless in a flat gravitation-free space. Such considerations have been discussed in the literature under the term "Einstein's elevator".

There is no doubt that there has been no change in the curvature of space due to the motion of the free-falling observers. $k=0$ does not mean that the underlying space is globally flat, but rather that it is only locally flat for the free-falling observers. This consideration is missing in papers which deal with cosmological models that expand in free fall.

\section{The Two Versions of the de Sitter Universe}

De Sitter designed a static cosmological model with a metric in the form (2.3). Its metric is of type $k=1$ and can be interpreted as a metric on a 4-dimensional pseudo-hypersphere embedded in a 5-dimensional flat space. The pseudo-hypersphere has the time-independent radius $R$. A transformation given by Lemaître [2] [3] transforms this metric into the form (2.4) with the scale factor $\mathrm{K}=\mathrm{e}^{\psi^{\prime}}$ via:

$$
\mathrm{d} s^{2}=\mathrm{K}^{2}\left[\mathrm{~d} r^{\prime 2}+r^{\prime 2} \mathrm{~d} \vartheta^{2}+r^{\prime 2} \sin ^{2} \vartheta \mathrm{d} \varphi^{2}\right]-\mathrm{d} t^{\prime 2}
$$

It is of type $k=0$. Other models, the anti-de-Sitter model, the Lanczos and the Lanczos-like model have similar characteristics. These models are grouped into the de Sitter family. The behavior of these models in transformations from comoving to non-comoving coordinates has been extensively studied by Florides [4]. We [5] [6] [7] have complemented the Lemaître coordinate transformations using Lorentz transformations.

Since the scale factor over $t^{\prime}=R \psi^{\prime}$ is time-dependent, the dS metric is considered in the form (2.4) as the metric of an expanding universe. However, this interpretation leads to contradictions. First of all, this view violates the principles of the general theory of relativity: A coordinate transformation cannot change the physical content of a theory. All possible coordinate systems are equal, and the choice of a particular coordinate system is usually a matter of utility.

The conservation law leads to another discrepancy. If one has redefined the cosmological constant that is unpopular with many authors using $\lambda=3 / R^{2}$, $\kappa p=-3 / R^{2}, \kappa \mu_{0}=3 / R^{2}$, one realizes that the mass density $\mu_{0}$ of the universe is constant, despite assumed expansion. Some authors have therefore tried to explain the constant mass density by producing new mass. However, this approach has proved unsatisfactory. Furthermore, Mitra [8] pointed out that, due to the equation of state $p+\mu_{0}=0$, no matter flow and no energy transport can be detected, even for the non-comoving observer.

The possibility of assigning a Lorentz transformation to the Lemaître coordinate transformation sheds some light on the problem [5] [6]. A Lorentz transformation transforms the static observer system into an accelerated one. In the static system, there are forces at every point in the cosmos that want to move the observers apart in all directions. The comoving observer system now follows 
these forces in free fall. According to Einstein's elevator principle [1], these forces are no longer perceptible in the comoving system. Instead of said forces, tidal forces [9] [10] occur. Mathematically, this process is carried out via the inhomogeneous transformation law of the Ricci-rotation coefficients.

This also makes it clear that neither a coordinate transformation nor a Lorentz transformation can change the geometric base structure of the space. $k=0$ in (3.1) thus results from Einstein's elevator principle and cannot be considered as a criterion for the flatness of the space. The question of how the de Sitter model is to be understood in its two versions was widely discussed among German physicists at the time. Finally, they turned to the great mathematician Klein [11]. His detailed answer ended the discussion. It is not known whether Klein's authority or the argumentative content of his work was the decisive factor. However, we cannot find any link between the geometries of the hyperspheres or their space-time slices and Klein's statements. We have not found any work that responds to Klein's publication.

The geometric structure of the pseudo-hypersphere may best expressed with the metric in the form of

$$
\begin{aligned}
& \mathrm{d} s^{2}=R^{2} \mathrm{~d} \eta^{2}+R^{2} \sin ^{2} \eta \mathrm{d} \vartheta^{2}+R^{2} \sin ^{2} \eta \sin ^{2} \vartheta \mathrm{d} \varphi^{2}+R^{2} \cos ^{2} \eta \mathrm{d} i \psi^{2} \\
& r=R \sin \eta, \quad R \mathrm{~d} i \psi=i \mathrm{~d} t .
\end{aligned}
$$

From it one takes the differential of the proper time

$$
i \mathrm{~d} T=\mathrm{R} \cos \eta \mathrm{d} i \psi, \quad \cos \eta=\sqrt{1-r^{2} / \mathrm{R}^{2}} .
$$

Parallel slices through the pseudo-hypersphere at an arbitrary position $r$ generate pseudo-circles (hyperbolae of constant curvature) with the $r$-dependent radii $R \cos \eta$ and the imaginary angle $i \psi$. The pseudo-circles are open, they range from $-\infty$ to $+\infty$ and have the same curvature everywhere, even at infinity. The pseudo-circle is drawn in the literature as a hyperbola in pseudo-real representation, which visualizes the $\mathrm{dS}$ cosmos as a one-shell hyperboloid. This has the advantage that the infinity of the timelines is recognizable. However, taking this representation literally can lead to errors. No hyperbolic property is recognizable in the $\mathrm{dS}$ model, no slice through the pseudo-hypersphere leads to a hyperbola.

At the point $r=\mathbb{R}$, the equator of the pseudo-hypersphere, $R \cos \eta=0$ and the pseudo-circle degenerates to a point in the pseudo-real representation. No time passes there, just as time stops at the event horizon of the Schwarzschild field. As can be seen from (3.3), this point lies at $r=\mathbb{R}$, i.e. at the equatorial spherical surface of the 3-dimensional hypersphere, which is assigned to an observer at an arbitrarily chosen pole. We call this area the geometric horizon.

The above-mentioned Lorentz transformation is associated with the Lemaitre transformation. From it, the relative speed of the observers, which are driven apart by the forces of the universe, can be read from

$$
v=\sin \eta=\frac{r}{R} .
$$


Thus, the relative velocity is geometrically determined. At a pole arbitrarily fixed with $r=0$, it has the value $v=0$ and, on the equatorial spherical surface, the value $v=1$, which is the value of the speed of light in the natural system of measurement. Thus, this horizon is also a cosmic horizon. In [12], we have shown that the observers' recession velocity can only reach the speed of light asymptotically. This means that in the dS universe, the basic laws of special relativity are not violated.

\section{The Subluminal Model}

The dS universe dealt with in the last section is not particularly suitable for the adaptation of astrophysical data. Nevertheless, it is significant for historical reasons. It has been instrumental in driving research into expanding cosmological models and is still the starting model for new expanding approaches. It has also been criticized that in expanding universes whose metric is known in comoving systems and to which a mass distribution can be assigned, no forces are acting on the masses. The expansion in free fall is responsible for the missing forces and consequently the common cosmic time for all observers. In [12], we envisaged an extended dS model in which the observers drift apart more slowly than in free fall and recognized forces acting on such observers. This model is only of mathematical importance, but the presented technique may be useful for building more sophisticated models.

Another, rather promising attempt was a model [13] that builds on the dS universe, but drops the condition $R=$ const. . We have called it a subluminal model because it definitively rules out that the recession velocity of the galaxies exceeds the speed of light. The subluminal universe is positively curved and closed. It has the position-independent pressure $\kappa p=-1 / R^{2}$ and the time-dependent mass density $\kappa \mu_{0}=3 / R^{2}$ with the equation of state $\mu_{0}+3 p=0$. Pressure and mass density result from the exact solutions of Einstein's field equations. The subluminal model therefore differs significantly from the FRW standard model in which the pressure is inserted by hand and is therefore not an exact solution to Einstein's field equations. Since the Einstein field equations do not fully determine FRW models, it is necessary to introduce numerous parameters, namely, the $\Omega$ s and the deceleration parameter. These quantities must then be filled using astrophysical data. The subluminal model needs only one parameter, the radius of curvature of the universe, or the scale factor. The Friedman equation takes the simple form $R^{*}=1, R^{*}=0$. The expansion rate of the model is constant.

For models that build on a pseudo-hypersphere with a non-constant radius, Einstein's field equations are insufficient to determine all the quantities of the model. The metric on a surface will determine the properties of that surface, but it will not be able to predict the change in the curvature of that surface. This is what the contracted Bianchi identities $R_{[m n \cdot r . \| s]}^{s}=0$ provide. They describe possible changes of the Riemann curvature tensor. For a genuine expanding cosmological model, two differential equation systems are needed 
The system (II) leads to the conservation law $T_{m \| n}{ }_{\| n}=0$. This is often used in the literature to establish an outstanding relation to variables. However, little reference is made to the above considerations. For models with constant $R$, the conservation law is trivial. Therefore, there is no need to use the system (II) to complement such a model.

The subluminal model has a geometric horizon, namely the equatorial surface of the hypersphere. As with the dS universe, it is determined by the relation (3.4) and, at the same time, it is the cosmic horizon. No galaxy can exceed the speed of light; it can only reach it asymptotically. Therefore, a galactic island formation is excluded. The possibility that superluminal speeds can occur has been deduced from Hubble's law. Using the redshift, it describes a linear relation between the recession velocity of the galaxies and the distance to a dislocated observer. However, this relation only allows for superluminal speeds if one assumes that in the Hubble equation $v=H r$ the variable $r$ is unbounded. Arbitrary distances are only possible in open infinite universes. In a closed universe with a geometrical horizon, the radial variable can only take the amount $R$, the radius of the universe, as the highest value.

We favor the view that infinite universes, be they flat or open, negatively curved ones, are ruled out as a way of describing Nature, this is because, on the one hand, infinities are hard to imagine, and on the other hand because we want to avoid conclusions from Hubble's law, which lead to acausalities and contradictions to the special theory of relativity.

Attempts have also been made to avoid the disagreeable implications of Hubble's law by arguing that the Hubble velocity is a coordinate speed that does not make reliable predictions. This problem does not apply to the subluminal model. If one differentiates $r=R \sin \eta$ according to cosmic time, one first obtains the non-invariant expression $r^{*}=\frac{R^{*}}{R} r$, which reduces to $R^{*}=1$ due to $v=r^{\cdot}=r / R=\sin \eta$. However, we have shown in [13] that this expression can be translated to $v=\mathrm{d} x^{1} / \mathrm{d} T$. Here, $\mathrm{d} x^{1}$ and $\mathrm{d} T$ are the proper length and proper time of a non-comoving observer. Thus, the recession velocity is defined independently of the coordinates and is also the velocity used in the Lorentz transformation, which transforms the non-comoving system into the comoving system.

In the introduction we explained, with the aid of the well-known Schwarzschild model, why gravity cannot be experienced in a free-falling elevator; we then transferred the problem to cosmic free-falling observers. We now want to address the problem in greater mathematical depth by borrowing a quantity from the Ricci-rotation coefficients that is closely related to the curvature of the space-like greater circles of the pseudo-hypersphere. 
The static dS metric is of type (2.3) and is the seed metric for the subluminal model. From this metric, using the standard technique of the tetrad representation, we obtain the above-considered quantity

$$
B_{m}=\left\{\frac{1}{r} \cos \eta, 0,0,0\right\}, \quad m=1,2,3,4 .
$$

Here $\eta$ is the polar angle of the pseudo-hypersphere and $\cos \eta=\sqrt{1-r^{2} / R^{2}}$. After a Lorentz transformation from the static system into the comoving system, this variable takes the form

$$
B_{m^{\prime}}=\left\{\alpha \frac{1}{r} \cos \eta, 0,0,-i \alpha \nu \frac{1}{r} \cos \eta\right\} .
$$

Here, according to (3.4), $v=\sin \eta$ is the relative velocity between the two systems and $\alpha=1 / \sqrt{1-\sin ^{2} \eta}=1 / \cos \eta$ is the assigned Lorentz factor. Finally, we have

$$
B_{m^{\prime}}=\left\{\frac{1}{r}, 0,0,-\frac{i}{R}\right\} .
$$

The spatial part of the quantity $B$ is $B_{\alpha^{\prime}}=\left\{\frac{1}{r}, 0,0\right\}, \alpha^{\prime}=1^{\prime}, 2^{\prime}, 3^{\prime}$ and corresponds to the expression of a flat geometric form. The same ${ }^{1}$ applies to the curvature of the space-like parallels of the pseudo-hypersphere

$$
C_{m^{\prime}}=\left\{\frac{1}{r}, \frac{1}{r} \cot \vartheta, 0,-\frac{i}{R}\right\}
$$

and for a time-like slice on the pseudo-hypersphere, so for a pseudo-circle

$$
U_{m}=\left\{U_{1}, 0,0,0\right\} \rightarrow{ }^{\prime} U_{m^{\prime}}=\left\{0,0,0,-\frac{i}{R}\right\} .
$$

From (4.3) it can be seen that even in the free-falling system, the space curvature is still present via the geometric term $\cos \eta$, but is compensated by the kinematic term $\alpha$. If one writes all components of the quantity $B$ in the 5-dimensional embedding space of the pseudo-hypersphere, one has with the local extra dimension $0^{\prime}$

$$
B_{a^{\prime}}=\left\{\frac{1}{r} \sin \eta, \alpha \frac{1}{r} \cos \eta, 0,0,-i \alpha v \frac{1}{r} \cos \eta\right\}, \quad a^{\prime}=0^{\prime}, 1^{\prime}, 2^{\prime}, 3^{\prime}, 4^{\prime} .
$$

This quantity can hardly be assigned to a flat space. Since all of the above expressions can be deduced directly from the type $k=0$ metric,

$$
\mathrm{d} s^{2}=\mathrm{K}^{2}\left(\mathrm{~d} r^{\prime 2}+r^{\prime 2} \mathrm{~d} \Omega^{2}\right)-\mathrm{d} t^{\prime 2}
$$

one will not be able to assume that $k=0$ inevitably leads to a flat space.

It should not be overlooked that after the Lorentz transformation, or, if one wishes, after the Lemaitre transformation, fourth components appear in the three basic quantities of the model, the sum of which produces the expansion scalar. To understand the meaning of these quantities, let us return to the ${ }^{1}$ Details on the calculation of Ricci-rotation coefficients can be found in our monographs [9] [10]. 
Schwarzschild model.

The new forces are the tidal forces, which act on the observer in Einstein's elevator but are too weak in Earth's proximity to be perceived by observers. Misner, Thorne, and Wheeler [14] derived these forces in their textbook with the aid of the geodesic deviation from the Riemann curvature tensor and Sharan [15] also illustrated them in his textbook. However, in an early article [16], we deduced the tidal forces directly from Einstein's field equations. We now want to transfer the process to the cosmological problem.

We summarize the three new components in (4.4), (4.5) and (4.6) to a quantity $^{2} D_{\alpha \beta}$

$$
D_{11}=D_{22}=D_{33}=-\frac{i}{R}, \quad D_{[\alpha \beta]}=0 .
$$

These are the gravitational forces that act on a freely expanding observer. As the space expands, they uniformly enlarge a volume around the observer in all three spatial directions. The Ricci-rotation coefficients are decomposed according to

$$
A_{m n}{ }^{s}=* B_{m n}{ }^{s}+{ }^{*} C_{m n}{ }^{s}+D_{m n}{ }^{s} .
$$

where $*_{B}$ and ${ }^{*} C$ are the spatial parts of $B$ and $C$. They appear to be flat, as stated above, and the subequations of Einstein's field equations drop out of Einstein's field equations with these quantities. As such, we only need to consider

$$
D_{m n}{ }^{s}=u_{n} D_{m}^{s}-u^{s} D_{m n}, \quad D_{s n}^{s}=u_{n} D_{s}^{s}, \quad u_{n}=\{0,0,0,1\} .
$$

The Ricci only contains relations with tidal forces:

$$
R_{m n}=-\left[D_{m n \wedge s} u^{s}+D_{m n} D_{s}^{s}\right]-2 u_{n} D_{[s \wedge \underline{m}]}^{s}-u_{m} u_{n}\left[D_{s \wedge r}^{s} u^{r}+D_{s r} D^{s r}\right] .
$$

Here $\underline{m}=1,2,3$ and $\Phi_{m \wedge n}=\Phi_{m \mid n}-{ }^{*} B_{n m}{ }^{s} \Phi_{s}-{ }^{*} C_{n m}{ }^{s} \Phi_{s}$ defines the space-like covariant derivative. The relation

$$
D_{[s \wedge \underline{s}]}^{s}=0
$$

indicates that the curvature of the model is location-independent and considerably simplifies the Ricci. The two remaining brackets in (4.12) lead to the results obtained using the Friedman equation. Of the Einstein field equations, only the relations

$$
\begin{aligned}
& R_{\alpha \beta}=-\left[D_{\alpha \beta \wedge s} u^{s}+D_{\alpha \beta} D_{\gamma}^{\gamma}\right], \quad{ }^{3} R=-\left[D_{\gamma}{ }_{\wedge s} u^{s}+D_{\delta}{ }^{\delta} D_{\gamma}^{\gamma}\right] \\
& R_{44}=-\left[D_{\gamma}{ }_{\wedge \wedge}{ }_{\wedge s} u^{s}+D_{\alpha \beta} D^{\alpha \beta}\right], \quad{ }^{4} R=-\left[D_{\gamma}{ }_{\wedge s}{ }_{\wedge s} u^{s}+D_{\alpha \beta} D^{\alpha \beta}\right]
\end{aligned}
$$

remain. After a short calculation, one obtains

$$
R_{\alpha \beta}=2 g_{\alpha \beta} \frac{1}{R^{2}}, \quad{ }^{3} R=\frac{6}{R^{2}}, \quad R_{44}=0, \quad{ }^{4} R=0 .
$$

We note that the 3-dimensional Ricci scalar ${ }^{3} R$ does not vanish. Finally, for the Einstein tensor one has

$$
G_{\alpha \beta}=-g_{\alpha \beta} \frac{1}{R^{2}}, \quad G_{44}=-\frac{3}{R^{2}}
$$

${ }^{2}$ Since we handle everything in the comoving system, we now omit the primes on the indices. 
and from that one obtains $\kappa p=-1 / R^{2}, \kappa \mu_{0}=3 / R^{2}$ and $\mu_{0}+3 p=0$, the results which are already known.

It can be seen from the above system of equations that curvature effects can also be described in the freely expanding system with the Einstein field equations.

\section{The Model of Melia}

In numerous papers ${ }^{3}$, Melia has proposed a cosmological model that is flat and infinite and thus also contains an infinite amount of matter. Matter, space, time, and infinity were thus created at the Big Bang. Melia has called his model the $R_{h}=c t$ model, where $R_{h}$ is the non-comoving radial coordinate at the cosmic horizon of the expanding model but $t$ is the cosmic time, i.e. the time in the system that comoves with the expansion.

A flat infinite model has no geometric horizon that defines the cosmic horizon. Melia, building on a flat universe, creates an event horizon by comparing it with the Schwarzschild theory. An enclosed mass $M=M\left(r_{h}\right)$ of a certain volume in the universe determines the Hubble radius ${ }^{4} r_{h}=2 G M / c^{2}$ and leads to the relation $r_{h}=c t^{\prime},\left(R_{h}=c t\right)$. The Hubble radius is the distance light has traveled since the Big Bang and $t^{\prime}$ the age of the universe. $r_{h}$ is the point at which the rate of expansion has reached the speed of light. From a point beyond $r_{h}$, there is no connection to an observer within $r_{h}$. Therefore, all considerations about whether Olbers' paradox can significantly influence the brightness of the sky of fixed stars are ruled out.

Since we believe that, despite the different view of the curvature parameter $k$, Melia's model is identical to our subluminal model, we have to show how Melia's quantities relate to our quantities. On the pseudo-hypersphere, $r=R \sin \eta$ applies with $R$ as the time-dependent radius of curvature and $\eta$ as the polar angle. At the equatorial surface, $\sin \eta=1$, thus

$$
r_{h}=R \text {. }
$$

This is the basic relation which connects the two models under discussion. The geometric horizon corresponds to the Hubble horizon and thus to Melia's event horizon. From $r_{h}=c t^{\prime}$ one immediately gains $R^{*}=c$ or in the natural measuring system

$$
R^{*}=1 \text {, }
$$

a relation that we obtained with the equation system II from (4.1) and which leads to the solution of the Friedman equation. Equation (5.2) accounts for the simplicity of the subluminal model. This model also has a geometric speed, like all models based on a concrete geometric form. It is, as already addressed in Sec. 3 ,

$$
v=\sin \eta=\frac{r}{\mathrm{R}}
$$

${ }^{3}$ Most papers by Melia and colleagues are listed in [13].

${ }^{4}$ Melia's variables $R, t$ correspond to our variables $r, t^{\prime}$, as we have used in earlier papers. 
and is the recession velocity of galaxies. For $r=r_{h}$, one has $v_{h}=1$. The recession velocity has reached the speed of light (asymptotically) at the equator. Higher speeds than the speed of light do not occur in either model, so the fundamental laws of special relativity remain.

To examine the relationship between the two models in greater depth, let us return to the above-mentioned definition of Melia's cosmic horizon. With respect to the cosmological principle of Weyl and the Birkhoff theorem, Melia determines the Hubble radius with

$$
r_{h}=\frac{2 G M\left(r_{h}\right)}{c^{2}} .
$$

Here,

$$
M\left(r_{h}\right)=\frac{4 \pi}{3} \frac{r_{h}^{3}}{c^{2}} \mu_{0}
$$

is the mass enclosed by the sphere with the radius $r_{h}$ and $\mu_{0}$ is the assigned mass density. Thus, with the aid of (5.1)

$$
r_{h}=\sqrt{\frac{3 c^{4}}{8 \pi G \mu_{0}}}=\sqrt{\frac{3}{\kappa \mu_{0}}}=\mathbb{R} .
$$

This immediately results in

$$
\kappa \mu_{0}=\frac{3}{R^{2}} .
$$

The mass density decreases as the universe increases in the radius $R$. These and similar relations can also be found in the Einstein universe, Friedman universe and the models of the $\mathrm{dS}$ family. However, this relation is not evident in the $R_{h}=c t$ model.

In addition, the discussion of whether the velocity defined by (5.3) is an invariant expression or a coordinate velocity remains brief. As was already explained in Sec. 4, (5.3) can be reduced to the invariant relation $v=\mathrm{dx} x^{1} / \mathrm{d} T$, with the de Sitter proper length $\mathrm{d} x^{1}$ and the proper time $\mathrm{d} T$ in the non-comoving system.

Both models describe the relation between the non-comoving radial coordinate $r$ and the comoving $r^{\prime}$ with

$$
r=K\left(t^{\prime}\right) r^{\prime},
$$

where $K$ is the time-dependent scale factor. We still have to show that the $R_{h}=c t$ model is compatible with the curvature of the pseudo-hypersphere. With

$$
r=R \sin \eta, \quad r^{\prime}=R_{0} \sin \eta, \quad R=K R_{0}, \quad R_{0}=\text { const. }
$$

we can write the Hubble parameter with both the scale factor and the pseudo-hypersphere's radius of curvature

$$
H=\frac{R^{*}}{R}=\frac{R^{*}}{R} .
$$


$R_{0}$ is the radius of curvature of the pseudo-hypersphere, if it is measured with the aid of comoving, i.e. expanding rods and therefore appears to be a constant quantity for the comoving observer.

Lastly, it would still be necessary to investigate whether the evaluation of Einstein's field equations results in different criteria for space curvature. Melia essentially relies on the Friedman equation; however, this is only one part of Einstein's field equations, namely the 44-components of Einstein's field equations. We did not find the remaining three subequations of Einstein's field equations in his papers. But they are precisely what is needed to provide information about the curvature structure of space. Two methods are available for working through the problem: the coordinate method with the Christoffel symbols $\Gamma$ as connexion coefficients and the tetrad formalism with the Ricci-rotation coefficients. Both types of coefficients depend on the relation

$$
A_{n m}^{s}=e_{n}^{k} \underset{m}{e_{i}^{i}} e_{j}^{s} \Gamma_{k i}^{j}+\stackrel{s}{e}_{j} e_{m \mid n}^{j} .
$$

The Ricci-rotation coefficients yield three quantities, the curvatures of the greater circles, parallels, and pseudo-circles on the pseudo-hypersphere. The Christoffel symbols provide a larger number of components, most of which contain a collection of trigonometric functions that can only be assigned very indirectly or perhaps not at all to geometric objects. The procedure is therefore not particularly suited for clarifying the question of whether $k=0$ must lead to a flat space.

Unless new arguments are submitted later that indicate a global flat space of the $R_{h}=c t$ model, the current situation is to be interpreted in such a way that both models, the $R_{h}=c t$ model and the subluminal model, are positively curved and therefore identical.

Melia has an extensive set of astrophysical data and has shown in some articles that this data can be best adapted to the $R_{h}=c t$ model, much better than to other FRW models. Thus, our subluminal model is well supported by Melia's data and analyses.

When developing our model, we did not envisage finding a model that closely relates to astrophysical data. Our goal was to provide mathematical foundations for a model that

1) is an exact solution to Einstein's field equations,

2) involves pressure, which is a result of this exact solution and is not inserted by hand, as is the case with numerous models,

3) does not allow superluminal speed and,

4) can be fully described geometrically.

The fact that this model is supported by astrophysical data was initially surprising to us, but it justifies our efforts. However, Melia's model also has an additional mathematical profile due to the subluminal model.

\section{Coordinate Systems}

Most cosmological models assume a metric written in comoving coordinates. 
This metric is also the natural framework for a model, because the rods and clocks associated with such a system are the ones we currently have available. Nevertheless, there is a need to present the obtained model in non-comoving coordinates as well. Of course, the question remains as to which new insights can be gained when looking for new representations. If one processes a model in tetrad calculus, a single coordinate system is sufficient to carry out operations such as differentiation and integration. Different coordinate systems are generally useful, but are of essentially equal value for certain problems. Comoving observers are characterized by $r^{\prime}=$ const., non-comoving observers by $r=$ const. . The question is how to realize the latter in practice. The position of such an observer must be continuously recalculated and a fixation to the calculated point in space requires significant technical effort.

It is preferable to search for non-comoving coordinates if one does not have a static reference system, because one does not know the Lorentz transformation which converts expanding systems into static ones. This is the case if the model does not provide geometric velocities or if one has not fully exploited the geometry.

If one has successfully set up an expanding model and knows the metric in comoving coordinates, one also has the corresponding tetrads $\stackrel{m}{e}_{i^{\prime}}$. If one also knows the geometrical speed or has determined the recession velocity in another way, one can also adjust the Lorentz transformation into a non-comoving system. With this and the inhomogeneous law of transformation of the Ricci-rotation coefficients, all field quantities can be calculated in the non-comoving system. With these, one can set up the stress-energy-momentum tensor and the conservation law and recalculate the field equations. These operations can all be done without the explicit use of a coordinate system.

However, if one wants to immediately start with a static system, the following possibility is a viable option: First of all, one transforms the expanding 4-bein $\left(m^{\prime}\right)$ with a Lorentz transformation into a static $(m)$

$$
e_{i^{\prime}}^{m}=L_{m^{\prime}}^{m} e_{i^{\prime}}^{m^{\prime}} .
$$

This would be enough to calculate the Ricci-rotation coefficients for the non-comoving system. However, it is also quite inconvenient, since the new tetrads are still indicated in the comoving coordinate system $(i ')$. Now the question arises as to whether the new 4-bein system can be diagonalized with a coordinate transformation $\Lambda$ with

$$
\stackrel{m}{e_{i}}=L_{m^{\prime}}^{m} e_{i^{\prime}}^{m^{\prime}} \Lambda_{i}^{i^{\prime}} .
$$

If, with a little intuition, one has found $\Lambda$, it must be ascertained whether this transformation is holonomic, i.e. whether it relates to coordinate lines. Thus, the relations

$$
\Lambda_{[i \mid k]}^{i^{\prime}}=0 \Rightarrow \Lambda_{i}^{i^{\prime}}=x^{i^{\prime}}{ }_{\mid i}
$$

must apply. That this need not always be the case has been demonstrated by a 
generalized dS model [12]. $\Lambda$ s can indeed be found for this model. However, these do not fulfil the relations (6.2). Therefore, the coordinates are anholonomic, meaning that there are no coordinate lines. The Ricci-rotation coefficients can therefore not be calculated with the 4-bein alone, but must be complemented by the object of the anholonomity

$$
\text { ' } A_{m^{\prime} n^{\prime}}{ }^{s^{\prime}}=A_{m^{\prime} n^{\prime}}{ }^{s^{\prime}}+\Lambda_{m^{\prime} n^{\prime}}{ }^{s^{\prime}}+\Lambda_{m^{\prime} n^{\prime}}^{s^{\prime}}+\Lambda_{n^{\prime} m^{\prime}}^{s^{\prime}}, \quad \Lambda_{m^{\prime} n^{\prime}}{ }^{s^{\prime}}=\underset{m^{\prime}}{e_{n^{\prime}}^{i^{\prime}}} e_{j^{\prime}}^{k^{\prime}} e_{j}^{s^{\prime}} \Lambda_{j}^{j^{\prime}} \Lambda_{\left[k^{\prime} \mid i^{i}\right]}^{j} .
$$

This of course questions the usefulness of the method.

The subluminal model provides a geometric velocity, with which the Lorentz matrix can be formed. With it, all field quantities can be transformed into the non-comoving system [13]. In particular, with the aid of the transformation analogous to (4.6) one has derived the radial force

$$
\begin{aligned}
& U_{m}=\hat{U}_{m}+f_{m}, \quad \hat{U}_{m}=\left\{-\alpha v \frac{1}{R}, 0,0,0\right\}, \quad f_{m}=\left\{i \alpha^{2} v \widetilde{F}_{4}, 0,0,-i \alpha^{2} v \widetilde{F}_{1}\right\} \\
& \widetilde{F}_{m^{\prime}}=\left\{0,0,0,-\frac{i}{R}\right\}, \quad \widetilde{F}_{m}=L_{m}^{m^{\prime}} \widetilde{F}_{m^{\prime}}=\left\{-\alpha v \frac{1}{R}, 0,0,-\alpha \frac{i}{R}\right\}
\end{aligned}
$$

which one is accustomed to derive from $g_{44}$ or $\stackrel{4}{e}_{4}$. Since $U_{1}$ is not a gradient, it is not possible to go in the opposite direction and derive the metric coefficient $g_{44}$ from (6.4). The quantity $F_{m}$ prevents this from being possible, wherein said quantity was obtained from the expansion of the universe. It is only if one switches off the expansion $(\tilde{F}=0)$ that the whole expression is reduced to the known dS quantity $\hat{U}$, which can be derived from $g_{44}$. Thus, to a non-comoving observer cannot be assigned a fully non-comoving coordinate system. It should also be remembered that in (6.4) we are looking for the quantity $g_{44}$, which is a solution to the differential equation system I. However, the expression containing the quantity $\widetilde{F}$ is a solution to the differential equation system II.

The search for the lapse function $g_{44}$ is probably historical. Even in the Schwarzschild model, the metric coefficients $g_{44}$ were used to calculate the gravitational redshift and/or time dilation. Recalling our discussion of free fall in the Schwarzschild field, we find that the ratio of the proper time of the free-falling observer and that of the static observer

$$
\frac{\mathrm{d} T^{\prime}}{\mathrm{d} T}=\frac{1}{\alpha}=\sqrt{1-\frac{2 M}{r}}
$$

is identical to $\sqrt{g_{44}}$. The time dilation can thus be deduced from the transformation behavior of the two observer systems. This applies equally to cosmological problems. For the subluminal model, one has

$$
\frac{\mathrm{d} T^{\prime}}{\mathrm{d} T}=\frac{1}{\alpha}=\sqrt{1-\frac{r^{2}}{\mathrm{R}^{2}}} .
$$

In this model, there is no need to resort to a possibly existing metric component $g_{44}$. If a model has a geometric velocity, the Lorentz factor can be created for observer systems moving relatively to each other and thus determine the redshift 
as a function of $r$.

No general method is known from the literature with which one could determine for which model static coordinates are possible. Investigations in this direction have been undertaken by Mitra [17] and Gautreau [18] [19], among others. In his papers, Melia has also tried to bring FRW metrics into the Schwarzschild form.

Apart from some marginal notes, we cannot contribute anything to this. It could be that Florides [4], with his six models, has already exhausted all the possibilities.

The subluminal model was developed by the simple generalization $R=R\left(t^{\prime}\right)$ from the $\mathrm{dS}$ model. The subluminal model therefore consists of a set of self-similar dS universes dislocated in the 5-dimensional space. The question thus arises as to whether these universes, together with time, can be covered by a single coordinate system.

On the other hand, one tries to set up a metric for a surface in non-comoving coordinates which describes not only the properties of the surface but also the temporal change of this surface. This attempt is reminiscent of the German story of Baron Münchhausen, who pulls himself out of the swamp by his own braid. The properties of the surface would have to be separated here, distinguishing between those belonging to system I and those belonging to system II.

\section{Conclusion}

In this paper, we have tried to establish a connection between our subluminal model and Melia's $R_{h}=c t$ model. We have argued that a cosmological metric with the curvature parameter $k=0$ does not necessarily require global flatness of the universe, but rather a local flatness due to the free fall of the expanding universe. We have confirmed our point of view by gradually introducing curvature variables into the $R_{h}=c t$ model, bringing the $R_{h}=c t$ model into the formal vicinity of the subluminal model. The identity of both models is thus ensured.

\section{Conflicts of Interest}

The author declares no conflicts of interest regarding the publication of this paper.

\section{References}

[1] Burghardt, R. (2016) Journal of Modern Physics, 7, 2347-2356. https://doi.org/10.4236/jmp.2016.716203

[2] Lemaitre, G. (1933) Annales de la Société Scientifique de Bruxelles A, 53, 51-85.

[3] Lemaître, G. (1997) General Relativity and Gravitation, 29, 641-680. https://doi.org/10.1023/A:1018855621348

[4] Florides, P.S. (1980) General Relativity and Gravitation, 12, 563-574. https://doi.org/10.1007/BF00756530 
[5] Burghardt, R. (2016) Transformations in de Sitter and Lanczos Models I. Austrian Reports on Gravitation. http://arg.or.at/Wpdf/WTrans1.pdf

[6] Burghardt, R. (2016) Transformations in de Sitter and Lanczos Models II. Austrian Reports on Gravitation. http://arg.or.at/Wpdf/WTrans2.pdf

[7] Burghardt, R. (2016) Transformations in de Sitter and Lanczos Models III. Austrian Reports on Gravitation. http://arg.or.at/Wpdf/WTrans3.pdf

[8] Mitra, A. (2015) Journal of Mathematical Physics D, 24, Article ID: 155002. https://doi.org/10.1142/S0218271815500327

[9] Burghardt, R. (2016) Spacetime Curvature. 1-597. http://arg.or.at/EMono.htm

[10] Burghardt, R. (2016) Raumkrümmung. 1-623. http://arg.or.at/Mono.htm

[11] Klein, F. (1918) Nachrichten von der Gesellschaft der Wissenschaften zu Göttingen, No. 3, 394-423.

[12] Burghardt, R. (2018) Journal of Modern Physics, 9, 685-700. https://doi.org/10.4236/jmp.2018.94047

[13] Burghardt, R. (2017) Journal of Modern Physics, 8, 583-601. https://doi.org/10.4236/jmp.2017.84039

[14] Misner, C.W., Thorne, K.S. and Wheeler, J.A. (1973) Gravitation. San Francisco.

[15] Sharan, P. (2009) Spacetime, Geometry and Gravitation. Progress in Mathematical Physics Vol. 56. Birkhäuser, Basel.

[16] Burghardt, R. (1995) Foundations of Physics Letters, 8, 575-582. https://doi.org/10.1007/BF02186248

[17] Mitra, A. (2013) Gravitation and Cosmology, 19, 134-137. https://doi.org/10.1134/S0202289313020072

[18] Gautreau, R. (1984) Physical Review D, 29, 186-197. https://doi.org/10.1103/PhysRevD.29.186

[19] Gautreau, R. (1983) Physical Review D, 27, 764-778. https://doi.org/10.1103/PhysRevD.27.764 